\title{
Robust charge stripe order under high electric fields in $\mathrm{Nd}_{1.67} \mathrm{Sr}_{0.33} \mathrm{NiO}_{4}$
}

\author{
M. Hücker ${ }^{1}$, M. v. Zimmermann ${ }^{2}$, G. D. $\mathrm{Gu}^{1}$ \\ ${ }^{1}$ Condensed Matter Physics and Materials Science Department, \\ Brookhaven National Laboratory, Upton, New York 11973, USA and \\ ${ }^{2}$ Hamburger Synchrotronstrahlungslabor HASYLAB at Deutsches Elektronen-Synchrotron DESY, 22603 Hamburg, Germany
}

(Dated: September 5, 2017)

\begin{abstract}
The influence of high electric fields on the charge stripe order in $\mathrm{Nd}_{1.67} \mathrm{Sr}_{0.33} \mathrm{NiO}_{4}$ was studied by means of simultaneous hard x-ray diffraction and electrical transport experiments. Direct measurements of the charge stripe satellite peaks in zero and high electric fields provide no evidence for a deformation or a sliding of the stripe lattice, which contradicts previous indications from non-linear conductance effects. By using the order parameter of a structural phase transition for instant sample temperature measurements, non-linear transport effects can be attributed to resistive heating. Implications for the pinning of stripes in the nickelates are discussed.
\end{abstract}

PACS numbers: $61.10 . \mathrm{Nz}, 71.45 . \mathrm{Lr}, 72.20 . \mathrm{Ht}, 74.72 . \mathrm{h}$

Electronic ground states with a modulated charge density are a common feature of correlated electron systems 1.2 One such class of materials is represented by the cuprate high temperature superconductors and isostructural nickelates, such as $\mathrm{La}_{2-x} \mathrm{Ba}_{x} \mathrm{CuO}_{4}$ and $\mathrm{La}_{2-x} \mathrm{Sr}_{x} \mathrm{NiO}_{4}, \frac{3.4}{3}$ Undoped $(x=0)$ these layered transition-metal-oxides are antiferromagnetic (AF) insulators. When introducing holes into the $\mathrm{CuO}_{2}$ and $\mathrm{NiO}_{2}$ planes $(x>0)$, they become conducting and the commensurate $\mathrm{AF}$ order is destroyed 5.6 For increasing $x$ the holes eventually micro phase separate into charge stripes, which form antiphase boundaries between spin stripes 3.7 .8 .9 While there is growing evidence supporting the existence of stripes, their physical properties and their relevance for the superconductivity in the cuprates are still unclear. In this context, an important question is how charge stripes respond to an electric field $E$, whether they start to slide or give rise to non-Ohmic behavior, and several experimental ${ }^{10.11 .12 .13 .14}$ and theoretical ${ }^{15.16 .17 .18}$ studies have addressed this problem.

A class of correlated electron materials, where nonOhmic effects are clearly observed, are one dimensional (1D) charge density wave (CDW) systems, such as the transition metal chalcogenide $\mathrm{NbSe}_{3}$ and the blue bronze $\mathrm{K}_{0.3} \mathrm{MoO}_{3} \stackrel{2}{2}$ Here, CDW form as a consequence of Fermi surface nesting $\stackrel{2}{=}$ When the CDW is incommensurate to the lattice it is usually pinned by impurities or defects such as grain boundaries ${ }^{19.20} \mathrm{~A}$ commensurate CDW is expected to couple stronger to the lattice, although in many cases impurity pinning is still dominant.2.21 Above an electric threshold field $E_{\mathrm{th}}$, these systems show a strong decrease of the resistivity, associated with the sliding of a rigid CDW. The threshold $E_{\text {th }}$ varies between the materials and increases with increasing impurity concentration ${ }^{20}$ Typical values are between $1 \mathrm{mV} / \mathrm{cm}$ and a few $\mathrm{V} / \mathrm{cm}^{2}{ }^{2}$ To gain insight in the sliding process, in several studies x-rays were used to analyze the CDW satellite reflections as a function of an applied $E$ field 19.22 .23

In the case of the cuprates and nickelates, significant efforts have been made to find fingerprints of non-Ohmic transport 10.11 .12 For some systems the results seem to in- dicate a dielectric breakdown at much higher thresholds than in classical CDW materials 10.11 In the nickelates the satellite reflections associated with the charge stripe order are relatively strong. This motivated us to directly probe their response to high $E$ fields, and to draw a comparison to previously reported anomalous transport 10

Here, we present x-ray diffraction and electrical transport measurements on $\mathrm{Nd}_{1.67} \mathrm{Sr}_{0.33} \mathrm{NiO}_{4}$, which show that the charge stripes stay locked to the lattice up to $E$ fields on the order of $\mathrm{kV} / \mathrm{cm}$. Deviations from Ohm's law have been identified to result from resistive heating. Crucial to our experiment is the use of an internal sample-thermometer, which is given by the $T$ dependent super structure reflection of the low-temperature less-orthorhombic (LTLO) phase ${ }^{24}$ Results for continuous and pulsed $E$ fields are qualitatively the same.

Several bar shaped $\mathrm{Nd}_{1.67} \mathrm{Sr}_{0.33} \mathrm{NiO}_{4}$ single crystals were prepared with dimensions $0.3 \times 1.2 \times 0.55 \mathrm{~mm}^{3}$ along the $a, b$, and $c$ axis, using the notation of the lowtemperature orthorhombic (LTO) phase ${ }^{24}$ The in-plane resistivity $\rho$ in Fig. 1 was measured with the four probe method along the $1.2 \mathrm{~mm}$ long direction. Since the crystals are only weakly twinned, for $\sim 90 \%$ of the sample volume both $a$-axis and charge stripes are perpendicular to the $E$ field ${ }^{24}$ To measure $\rho$, at low $T$ (40-135 K) and high $E$ fields a Keithley 2410 source meter was used, and a Keithley 2000 otherwise. The leads were attached with silver epoxy, carefully cured to reduce the contact resistance. The sample was mounted on a cold-finger (CF) using heat conducting epoxy. At low $T$, currents $I \gtrsim 2 \mathrm{~mA}$ sometimes caused damage to the contacts, so that $I$ was usually limited to $I_{\text {limit }}=1-1.5 \mathrm{~mA}$. The x-ray studies were performed at beamline BW5 at HASYLAB. At a photon energy of $100 \mathrm{keV}$ and a beam diameter of $1 \times 1 \mathrm{~mm}^{2}$, the bulk properties of the stripe order and the crystal structure were studied in transmission geometry.

Figure 1 summarizes the structural and transport properties of $\mathrm{Nd}_{1.67} \mathrm{Sr}_{0.33} \mathrm{NiO}_{4}$ at zero and very low $E$ fields, respectively. Two structural transitions are observed. The LTO/LTLO transition at $T_{\mathrm{LT}} \sim 97 \mathrm{~K}$ is identified by the appearance of the $(1,1,0)$ reflection ${ }^{24}$ 


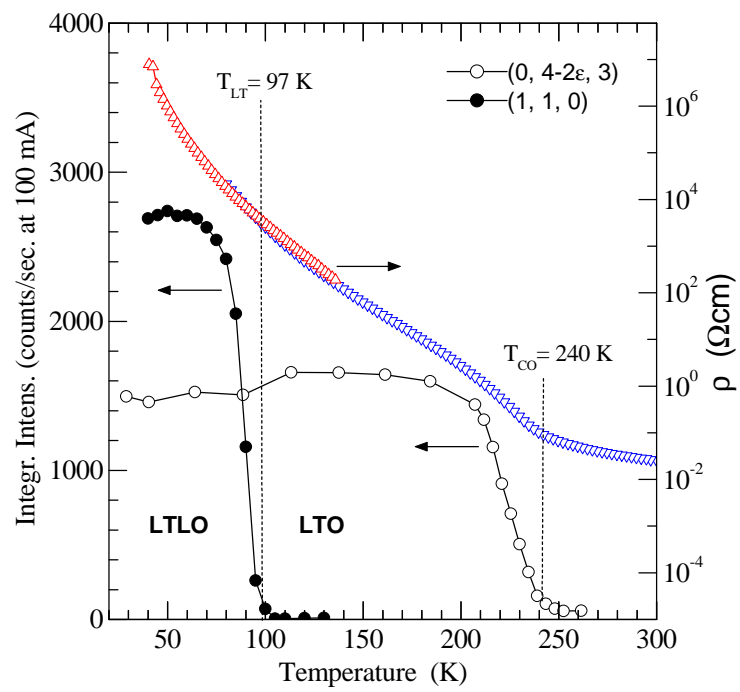

FIG. 1: (Color online) $T$ dependence of the in-plane resistivity $\rho$ as well as the integrated intensity of the charge stripe peak $(0,4-2 \epsilon, 3)$ and the super structure peak $(1,1,0)$.

Apparently, this peak shows a strong $T$ dependence between $60 \mathrm{~K}$ and $100 \mathrm{~K}$, which we have used as an internal sample thermometer. The charge stripe order below $\sim 240 \mathrm{~K}$ is indicated by satellite reflections with ordering wave vector $q_{C O}=(0,2 \epsilon, 2 / 3)$, where $\epsilon \simeq x=0.33$, and $1 /(2 \epsilon)$ corresponds to the in-plane stripe distance ${ }^{24}$ Figure 1 shows the integrated intensity from $l$ scans through the charge peak centered at $(0,4-2 \epsilon, 3)$. Below $\sim 180 \mathrm{~K}$ the intensity is constant, except for a step at $T_{\mathrm{LT}}$, which is also observed for fundamental reflections, and can be attributed to extinction effects. In $\rho$ the onset of charge stripe order is marked by a strong increase, whereas the LTO/LTLO transition leads to no signature (Fig. 1).

Next we turn towards the high $E$ field experiments. In Fig. 22(a) we show $I / E$-curves for different cold-finger temperatures $T_{\mathrm{CF}}$, where $E$ was measured as a function of a continuous current. The curves are non-linear and exhibit pronounced maxima. These maxima correspond to the thresholds $E_{\mathrm{th}}$ in an experiment were $I$ is measured as a function of $E$. For $I\left(E \ll E_{\mathrm{th}}\right)$ the slope $d E / d I$ corresponds to $\rho$ in Fig. 1 Simultaneous with the $I$ scans we have measured the $(1,1,0)$ peak intensity [Fig. 2(b)]. Apparently, the intensity decreases as $I$ increases, with the strongest change occurring at lower $I$ for higher $T_{\mathrm{CF}}$. Since the LTO/LTLO transition does not depend on current or voltage, this effect must have a different reason. To rule out experimental flaws, we have verified that the $(1,1,0)$ peak does not move and that all its intensity is integrated. We conclude that, with increasing $I$, the sample temperature $T_{S}$ has increased significantly above $T_{\mathrm{CF}}$. In particular, whenever the $(1,1,0)$ intensity approaches zero, $T_{S}$ has risen to above $T_{\mathrm{LT}} \sim 97 \mathrm{~K}$. According to Fig. 1 an increase of $T_{S}$ by several $10 \mathrm{~K}$ causes $\rho$ to drop by up to two orders of magnitude, which implies that the non-linear $I / E$-curves in Fig. 2(a) are evidence for thermal switching rather than a dielectric breakdown.

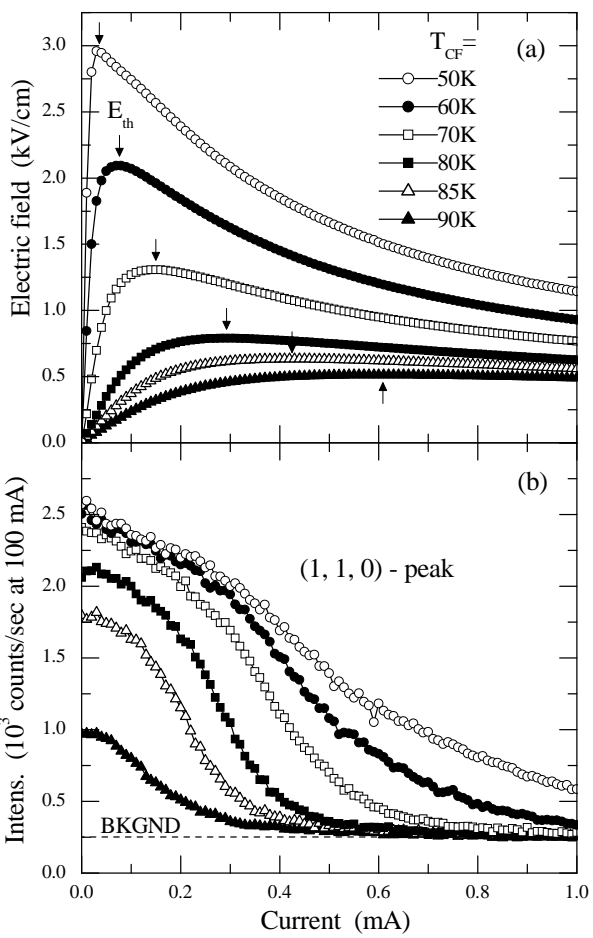

FIG. 2: Current dependence of (a) the applied in-plane field $E$ and (b) the $(1,1,0)$ peak intensity for different $T_{\mathrm{CF}}$. The arrows in (a) mark the threshold field.

Thermal switching (TS) depends mainly on the thermal coupling between sample and cold-finger, and the derivative $d \rho / d T, 12.25 .26 E_{\mathrm{th}}^{\mathrm{TS}}$ is reached when the resistive heating compensates the sample cooling. Once resistive heating dominates, thermal switching evolves the faster the larger $-d \rho / d T$. This effect is neither caused by, nor evidence for non-Ohmic transport. Similar conclusions have been reported for $\mathrm{La}_{2-x} \mathrm{Sr}_{x} \mathrm{CuO}_{4}$ thin films and $\mathrm{Pr}_{0.8} \mathrm{Ca}_{0.2} \mathrm{MnO}_{3}$ single crystals $\frac{12,27}{}$

To explore if the interfering sample heating can be avoided by means of short pulses, we have compared $T$ cycles for both pulsed $(200 \mathrm{msec})$ and continuous $E$ fields. The results in Fig. 3 show the current $I$ and the $(1,1,0)$ peak intensity as a function of $T_{\mathrm{CF}}$ for $E$ fields of $0.4 \mathrm{kV} / \mathrm{cm}, 0.8 \mathrm{kV} / \mathrm{cm}$, and $1.2 \mathrm{kV} / \mathrm{cm}$. As reference for the case of no sample heating, a zero field approximation for $I$ was extracted from $\rho(T)$ in Fig 1 For continuous $E$ and increasing $T_{\mathrm{CF}}$, a sudden increase of $I$ up to $I_{\text {limit }}$ is observed, indicating a drastic decrease of $\rho$. At the same time the $(1,1,0)$ peak intensity drops to zero [Figs. [3(a) and (b)]. This shows that thermal switching has taken place and that $T_{S}$ has jumped to a value above $T_{\mathrm{LT}}$. Obviously, the higher $E$ the lower the critical $T_{\mathrm{CF}}$ where thermal switching occurs, which is consistent with the result in Fig. 22 (a) that $E_{t h}^{\mathrm{TS}}$ decreases with increasing $T_{\mathrm{CF}}$.

Similar results are obtained in the pulsed mode, with 200 msec pulse length and a 2 sec gap between the pulses [Figs. 3(c) and (d)]. With increasing $T_{\mathrm{CF}}$ the measured I suddenly starts to increase significantly above the zero 


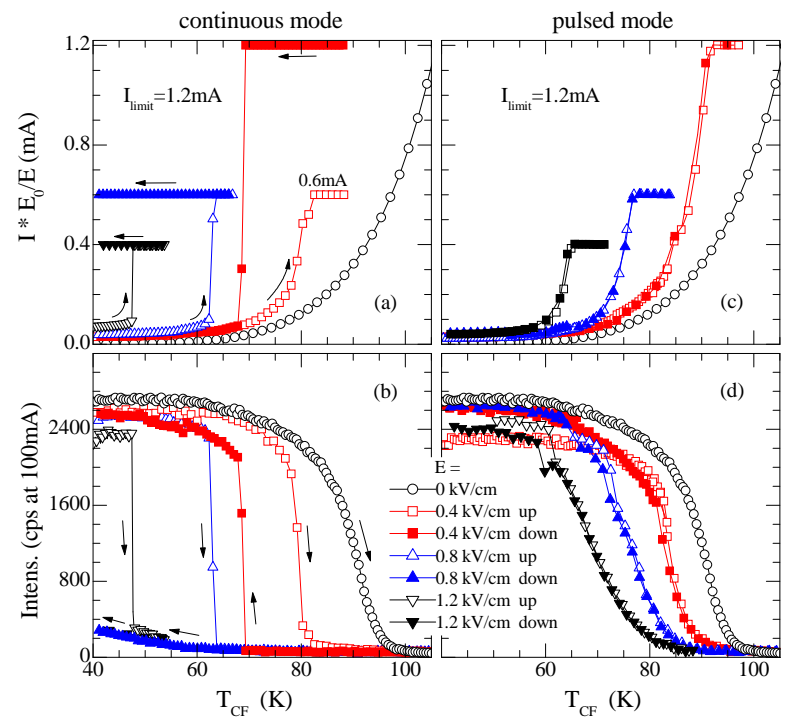

FIG. 3: (Color online) In-plane current normalized to $E_{0}=$ $0.4 \mathrm{kV} / \mathrm{cm}$, and the $(1,1,0)$ peak intensity vs $T_{\mathrm{CF}}$ for different $E$ fields. (a),(b) continuous $E$ field. (c),(d) pulsed $E$ field (200 ms). $I_{\text {limit }}$ is $1.2 \mathrm{~mA}(0.6 \mathrm{~mA}$ for $0.4 \mathrm{kV}$ up). $E$ values are valid for $I<I_{\text {limit }}$, only. As soon as $I=I_{\text {limit }}, E$ decreases with increasing $T_{\mathrm{CF}}$.

field reference, although not as sharply. Again this effect goes along with a decrease of the $(1,1,0)$ peak intensity, indicating significant sample heating. The power dissipated during a $200 \mathrm{msec}$ pulse is of course smaller than for continuous $E$, which shifts the thermal switching point to somewhat higher $T_{\mathrm{CF}}$. Note that photons where counted throughout the entire $200 \mathrm{msec}$ pulse, whereas $I$ was measured at its end. Therefore, Fig. 3(d) shows an average intensity, which is larger than the intensity one would measure during a very short time at the end of the pulse, if the count rates would allow one to do so. This explains why the transitions in $(1,1,0)$ are broader than for $I$ and slightly shifted to higher $T_{\mathrm{CF}}$. When accounting for this effect, the transitions in Figs. 3(c) and 3(d) become equally sharp and have the same critical $T_{\mathrm{CF}}$. For details see Ref. 26.

The continuous mode yields a large $T_{\mathrm{CF}}$ hysteresis, which is absent in the pulsed mode (Fig. 31). In the pulsed mode no hysteresis is expected, since the sample cools down to $T_{\mathrm{CF}}$ after each pulse; the conditions before a pulse are the same for the heating and the cooling cycles. In contrast, in the continuous mode the conditions before each pulse depend on the history. Once the sample has thermally switched into the low resistive state, $T_{\mathrm{CF}}$ must be decreased significantly before it can return to the high resistive state. Depending on $E$ and $I_{\text {limit }}$, this may not happen even for $T_{\mathrm{CF}}=0 \mathrm{~K}$. We conclude that there is no qualitative difference between the pulsed and the continuous modes. In both cases non-linear conductance effects are caused by sample heating. In the pulsed mode heating effects are lower, although $200 \mathrm{msec}$ pulses are still too long to reach significantly higher $E$ fields with-

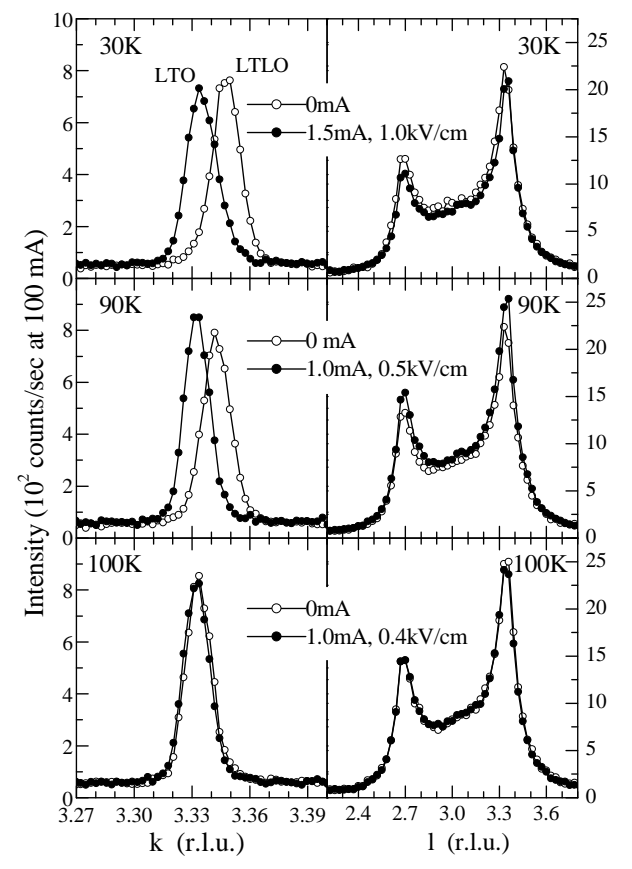

FIG. $4: k$ and $l$ scans through $(0,4-2 \epsilon, 3)$ charge peak at zero and finite continuous current. The peak shift observed in the $k$ scans is due to the contraction of the $b$ axis in the LTLO phase.

out causing a drastic sample heating during the pulse.

Although our results indicate that in $\mathrm{Nd}_{1.67} \mathrm{Sr}_{0.33} \mathrm{NiO}_{4}$ a high $E$ field causes mainly, if not exclusively, sample heating, it is still possible that it also affects the stripe order. Therefore, we have studied the shape and intensity of the $(0,4-2 \epsilon, 3)$ charge stripe peak for $I=0 \mathrm{~mA}$ and $1 \mathrm{~mA}\left(1.5 \mathrm{~mA}\right.$ for $\left.T_{\mathrm{CF}}=30 \mathrm{~K}\right)$, where the finite current is above the threshold for thermal switching. Figure 4 shows $k$ and $l$ scans for $T_{\mathrm{CF}}=30 \mathrm{~K}, 90 \mathrm{~K}$ and $100 \mathrm{~K}$. The double peak profile of the $l$ scans indicates a three-

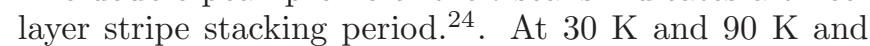
$I=0 \mathrm{~mA}$ the sample is in the LTLO phase. When turning on $I$, it transforms into the LTO phase, as is evident from the shift of the peak in $k \stackrel{24}{2}$ At $T_{\mathrm{CF}}=100 \mathrm{~K}$ the sample is in the LTO phase for both zero and finite $I$. Obviously, there are no significant differences in intensity or peak shape between zero and finite $I$ at any $T_{\mathrm{CF}}$. Both the in-plane order and the stacking of the stripes are unchanged. Thus, charge stripes in $\mathrm{Nd}_{1.67} \mathrm{Sr}_{0.33} \mathrm{NiO}_{4}$ are robust against $E$ fields on the order of $1 \mathrm{kV} / \mathrm{cm}$. Note that the slight intensity increase at $90 \mathrm{~K}$ and $1 \mathrm{~mA}$ is consistent with the extinction effects at the LTLO/LTO transition (cf. Fig. (1). At $T_{\mathrm{CF}}=30 \mathrm{~K}$ the slightly smaller intensity at $1.5 \mathrm{~mA}$ follows from a peak broadening in $k$, indicating that $T_{S}$ is inhomogeneous due to the large gradient between $T_{S}$ and $T_{\mathrm{CF}}$.

The results in Fig. 4 show that in the studied $T$ region for a depinning of stripes, if possible at all, higher $E$ fields have to be applied. This, however, will be difficult because the dissipated energy increases with $E^{2}$. As our results show, already for $\mathrm{kV} / \mathrm{cm}$ fields and $\mathrm{mA}$ 
currents sample heating is a serious problem (cf. discussion in Ref. 12). For millisecond pulse lengths $10 \mathrm{kV} / \mathrm{cm}$ may be reached at low $T$. However, on this time scale it is difficult to track stripe peak intensities, in addition to problems emerging from the electrical contacts and thermal stress on the sample.

It is obvious that the nickelates behave very different from the classical 1D-CDW materials. The stripes emerge from an electronic phase separation, primarily driven by $\mathrm{AF}$ correlations and Coulomb interactions $\frac{3}{\underline{3}} \mathrm{In}$ addition, Hund's-rule exchange causes a strong localization of the stripes, accompanied by significant local lattice distortions $\stackrel{28.29 .30}{ }$ Hence, each Ni site constitutes a pinning center. For this type of system it is not surprising that even for $\mathrm{kV} / \mathrm{cm}$ electric fields stripes do not slide. Impurities may cause some disorder, but most likely do not determine the order of magnitude of a dielectric threshold. Should a dielectric threshold $E_{t h}^{\mathrm{D}}$ exist, it may be unreachable with conventional $I / E$-experiments. Furthermore, it remains unclear whether stripes would slide as a whole, break up locally, or disintegrate as soon as $E_{t h}^{\mathrm{D}}$ is exceeded. The particular scenario depends on the stiffness of the sliding stripes compared to the pinning forces. Note that the in-plane correlations of the stripes are stronger than their interplane correlations. Therefore, we would expect that, with increasing $E$ field, first the three-layer stacking order degrades, resulting in a change of the double peak in the $l$ scans. As is evident from Fig. 4 this is not the case in our data.

In classical 1D-CDW systems the charge carriers have more itinerant character, and the electron-lattice coupling is weak. In this case the CDW usually is pinned by impurities, resulting in a collective mode in the optical conductivity at energies on the order of $\hbar \omega_{0} \sim$ $0.01-0.1 \mathrm{meV}$, which is well below the single particle gap energy $\stackrel{2}{\underline{2}}$ So far, there is no evidence pointing towards a similar mode in the nickelates ${ }^{31.32}$ Here, the most in- triguing feature, believed to be connected to stripe correlations, is the broad mid-gap infrared peak at $\sim 0.6 \mathrm{eV} \mathbf{s}^{32}$ This gap seems to roughly correspond to the activation energy of $\rho$, indicating that it is most likely associated with single particle excitations rather than collective excitations ${ }^{32}$ It also persists to temperatures above $T_{\mathrm{CO}}{ }^{31}$ In a simple model developed for pinned CDW, $E_{\mathrm{th}}^{D} \propto \omega_{0}^{2}{ }^{2.20}$ Assuming the case that the ratio $E_{\mathrm{th}}^{D} / \omega_{0}^{2}$ is the same for $1 \mathrm{D}-\mathrm{CDW}$ systems and stripes, this allows one to calculate some rough estimates. On one hand, plugging in $1 \mathrm{kV} / \mathrm{cm}$ gives a lower limit for the collective mode of about $\hbar \omega_{0} \sim 10 \mathrm{meV}$. Although this energy is at least two orders of magnitude larger than for the mentioned CDW systems, it covers only a small part of the energy window below the mid-gap peak. On the other hand, it is reasonable to assume that the energy of any collective mode in the nickelates will not exceed the midgap peak energy. Hence, with $\hbar \omega_{0} \sim 0.6 \mathrm{eV}$, the upper limit for $E_{t h}^{\mathrm{D}}$ easily enters the $10^{6}-10^{7} \mathrm{~V} / \mathrm{cm}$ range. Unless $E_{t h}^{\mathrm{D}}$ turns out to be several orders of magnitude smaller, macroscopic non-Ohmic transport in the stripe ordered nickelates may be out of reach.

In summary, we find that the static charge stripe order in $\mathrm{Nd}_{1.67} \mathrm{Sr}_{0.33} \mathrm{NiO}_{4}$ is stable in electric fields on the order of $\mathrm{kV} / \mathrm{cm}$. Neither the in-plane stripe order, nor the stacking order is affected. Non-linear conductivity effects, previously associated with a sliding of the stripes, are due to resistive sample heating. Our results are evidence of the strong charge carrier localization in stripe ordered nickelates, which distinguishes this class of materials dramatically from classical CDW systems, where the weakly pinned CDW emerges from Fermi surface nesting.

We would like to thank J. M. Tranquada, C. C. Homes and T. M. Rice for helpful discussions. The work at Brookhaven was supported by the Office of Science, U.S. Department of Energy under Contract No. DE-AC0298CH10886.
1 E. Dagotto, Science 309, 257 (2005).

2 G. Grüner, Density Waves in Solids volume 89 of Frontiers in Physics. Addison-Wesley Publishing Company 1994.

3 J. M. Tranquada et al., Nature 375, 561 (1995).

4 V.J. Emery and S.A. Kivelson, Physica C 209, 597 (1993).

5 M. A. Kastner and R. J. Birgeneau, Rev. Mod. Phys. 70, 897 (1998).

6 J. M. Tranquada, in Neutron Scattering in Layered CopperOxide Supercondcutors, edited by A. Furrer (Kluwer, Dordrecht, 1998), p. 225.

7 P. Abbamonte et al., Nature Physics 1, 155 (2005).

8 M. Fujita et al., Phys. Rev. B 70, 104517 (2004).

9 S. A. Kivelson et al., Rev. Mod. Phys. 75, 1201 (2003).

10 S. Yamanouchi et al., Phys. Rev. Lett. 83, 5555 (1999).

11 Y. Taguchi et al., Phys. Rev. B 62, 7015 (2000).

12 A. N. Lavrov et al., Phys. Rev. B 68, 94506 (2003).

13 G. Blumberg et al., Science 297, 584 (2002).

14 A. Asamitsu et al., Nature 388, 50 (1997).

15 C. Morais Smith et al., Phys. Rev. B 58, 453 (1998).
16 N. Hasselmann et al., Phys. Rev. Lett. 82, 2135 (1999).

17 S. Bogner and S. Scheidl, Phys. Rev. B 64, 54517 (2001).

18 T. Oka et al., Phys. Rev. Lett. 91, 66406 (2003).

19 Y. Li et al., Phys. Rev. Lett. 83, 3514 (1999).

20 D. Reagor and G. Grüner. Phys. Rev. B 39, 7626 (1989).

21 J. Dumas et al., Phys. Rev. Lett. 50, 757 (1983).

22 R. Danneau et al., Phys. Rev. Lett. 89, 106404 (2002).

23 H. Requardt et al., Phys. Rev. Lett. 80, 5631 (1998).

24 M. Hücker et al., Phys. Rev. B 74, 85112 (2006).

25 D.M. Kroll, Phys. Rev. B 9, 1669 (1974).

26 M. v. Zimmermann et al., (unbuplished).

27 S. Mercone et al., J. Appl. Phys. 98, 23911 (2005).

28 V. I. Anisimov et al., Phys. Rev. Lett. 68, 345 (1992).

29 J. Zaanen and P. B. Littlewood, Phys. Rev. B 50, 7222 (1994).

30 J. M. Tranquada, J. Phys. Chem. Solids 59, 2150 (1998).

31 T. Katsufuji et al., Phys. Rev. B 54, 14230 (1996).

32 C.C. Homes et al., Phys. Rev. B 67, 184516 (2003). 